Dom. Cien., ISSN: 2477-8818

Vol. 4, núm. 1, enero, 2018, pp. 65-79

Aproximación al uso de las redes sociales en internet desde una revisión teórica

\title{
Aproximación al uso de las redes sociales en internet desde una revisión teórica
}

\section{Approach to the use of social networks on the internet from a theoretical review}

\section{Abordagem do uso de redes sociais na internet a partir de uma revisão teórica}

\author{
Adriana V. Cadena-Silva ${ }^{\mathrm{I}}$ \\ vanecadena19@gmail.com \\ Sergio F. Mieles-Bachicoria ${ }^{\text {II }}$ \\ sergiomieles19@gmail.com \\ Janeth V. Intriago-Vera ${ }^{\text {III }}$ \\ tintriago@hotmail.com
}

Recibido: 23 de mayo de 2017 * Corregido: 18 de julio de 2017 * Aceptado: 27 de septiembre 2017

I. Magister en Docencia y Desarrollo del Currículo, Ingeniera en Sistemas, Docente de la Universidad Laica Eloy Alfaro de Manabí, El Carmen, Ecuador.

II. Magister en Docencia y Desarrollo del Currículo, Ingeniero en Sistemas, Docente de la Universidad Laica Eloy Alfaro de Manabí, El Carmen, Ecuador.

III. Magister En Gestión del Talento Humano, Ingeniera en Administración Pública, Tecnólogo en Administración Pública, Docente de la Universidad Laica Eloy Alfaro de Manabí, El Carmen, Ecuador. 
Dom. Cien., ISSN: 2477-8818

Vol. 4, núm. 1, enero, 2018, pp. 65-79

Aproximación al uso de las redes sociales en internet desde una revisión teórica

\section{Resumen}

Las redes sociales de Internet se han incorporado de manera importante a la vida de los seres humanos, de modo que se encuentran presentes prácticamente en todos los ámbitos. En este trabajo se expone el uso de las redes sociales en diferentes contextos: 1.- la comunicación interna de las organizaciones, 2.- la mercadotecnia, publicidad y comercio, 3.- la esfera cultural, y 4.- en el contexto educacional. Para ello se realiza un análisis documental a partir de las diferentes fuentes de información que aparecen en Internet.

Palabras clave: Internet; redes sociales; usos; web 2.0.

\section{Abstract}

The social networks of the Internet have been incorporated in an important way into the lives of human beings, so that they are present practically in all areas. This paper exposes the use of social networks in different contexts: 1.- the internal communication of the organizations, 2.- the marketing, advertising and commerce, 3.- the cultural sphere, and 4.- in the educational context. For this, a documentary analysis is made from the different sources of information that appear on the Internet. Keywords: Internet; social networks; applications; web 2.0.

\section{Resumo}

As redes sociais da Internet foram incorporadas de forma importante às vidas dos seres humanos, de modo que estejam presentes praticamente em todas as áreas. Este artigo expõe o uso de redes sociais em diferentes contextos: 1.- a comunicação interna das organizações, 2.- o marketing, a publicidade e o comércio, 3.- a esfera cultural e 4.- no contexto educacional. Para isso, uma análise documental é feita a partir das diferentes fontes de informação que aparecem na Internet.

Palavras chave: Internet; redes sociais; usa; web 2.0.

\section{Introducción}

El término Internet suele referirse a una interconexión en particular, de carácter planetario y abierto al público, que conecta redes informáticas de organismos oficiales, educativos y empresariales. 
También existen sistemas de redes más pequeños llamados Intranet, generalmente para el uso de una única organización.

A través de internet se accede a la World Wide Web (WEB), que es un sistema de documentos de hipertexto o hipermedios enlazados. Con un navegador web, un usuario visualiza sitios web compuestos de páginas web que pueden contener texto, imágenes, vídeos u otros contenidos multimedia, y navega a través de ellas usando hiperenlaces. (ECURED, 2017)

La Web ha evolucionado de aplicaciones básicas, tradicionales hacia aplicaciones web enfocadas al usuario final tales como las redes sociales. A esta actitud se le llama Web 2.0. Ahora bien si se parte de que el hombre nace en sociedad y en su desarrollo se integra a grupos con intereses afines y va formando un entramado de relaciones sociales con sus semejantes, es correcto entonces afirmar que las redes sociales no nacen con Internet. Un usuario de la red al conectarse a una red social, espera satisfacer necesidades de comunicación, con personas afines. (ECURED, 2017)

La Web 2.0 identifica el nivel de desarrollo socio-tecnológico de Internet; es decir, representa la evolución social de Internet y se acerca al escenario ideal donde la interacción social es capaz de dar soporte tecnológico al desarrollo de una comunidad virtual, considerada como el recurso activo de una auténtica sociedad de la información y el conocimiento. (Islas Torres C., 2011)

Area Moreira (2008) habla de tres tipos de redes sociales online:

- Redes de masas: son las más conocidas. Hablamos de Facebook, Twitter, Tuenti, Hi5... En ella los usuarios comparten un perfil, sus fotos, comentarios, etc.

- Redes abiertas para compartir archivos en distintos formatos. Youtube (para los vídeos), Flickr (para las fotografías), Slideshare (presentaciones), etc., son algunos de los miles software encontrados en la red.

- Redes temáticas o comunidades de aprendizaje con un objetivo común o interés específico. Hablamos de Ning, Elgg, Socialgo, etc. (Área Moreira M., 2008) 
Aproximación al uso de las redes sociales en internet desde una revisión teórica

Meso (2010), presentó datos relevantes de un estudio realizado en España, donde el éxito de las redes sociales ha sido motivo de debates en los círculos académicos, se dice que en los últimos dos años las redes se han convertido en la aplicación más exitosa de la sociedad de la información.

Evidentemente las redes sociales se han incorporado de manera importante a la vida de los seres humanos, de modo que se encuentran presentes prácticamente en todos los ámbitos. Este trabajo tiene como objetivo exponer el uso de las redes sociales en diferentes contextos.

Para ello se realiza un análisis documental a partir de las diferentes fuentes de información que aparecen en Internet.

\section{Desarrollo}

\section{El uso de las redes sociales en la comunicación interna de las organizaciones}

La comunicación interna debe ser una prioridad de la dirección en cualquier organización. El uso de las redes sociales en la empresa implica una mayor efectividad y una optimización mayor de las personas que trabajan con unos intereses comunes. Un empleado implicado en la consecución de los objetivos trabajará mucho más a gusto que un empleado que se siente desoído por su organización. (Cristófol F.J., 2016)

Las Redes Sociales se consolidan como un canal de comunicación interna en las empresas (...) De hecho, los empleados utilizan las redes sociales para aportar ideas sobre aspectos organizativos en el $56 \%$ de estas empresas, frente a un $41 \%$ que lo hacían el año pasado. Además, el 58\% de estas organizaciones utilizan las redes sociales para fomentar el contacto dentro de la organización, y el $85 \%$ ofrece foros o blogs para compartir el conocimiento o para alentar el contacto interno. (Torres N., 2014)

(...) Muchas organizaciones no ven con malos ojos incorporar las redes sociales como herramienta de mejora en la comunicación interna de las empresas. Claro que se trata de una red social de ámbito interno y corporativo. Nada que ver con Facebook. Pero incorpora la facilidad que nos da la red social 
Aproximación al uso de las redes sociales en internet desde una revisión teórica

para comunicarnos, crear grupos de trabajo, proponer debates, etc. Y todo ello dentro de nuestra empresa, donde en lugar de amigos, agregamos compañeros de trabajo. Y en lugar de enviar una cadena de correos electrónicos para discutir un asunto, podemos publicar en el tablero de un proyecto nuestras opiniones sobre cómo debe desarrollarse el mismo. Por ejemplo, para saber si alguien ha leído una circular, bastará con que marque el botón de leído (...) Si la empresa se encuentra dispersa la comunicación a través de las redes sociales facilita mucho el diálogo sin necesidad de añadir ruido a muchas cuentas. (PYMES, 2012)

En la literatura (PYMES, 2012) (Cristófol F.J., 2016) aparecen diferentes escenarios donde las redes sociales ayudan a la comunicación interna:

- Yammer, red social pensada para las empresas, ofrece una plataforma de colaboración y agilizar la comunicación entre los flujos de trabajo y las conversaciones en la red social.

- Google Apps, red social dentro de la propia organización que incorpora herramientas tales como videocoferencias, el correo de Gmail y Google Docs.

- Zyncro, alternativa de red social colaborativa en la nube que facilita la plataforma perfecta para la colaboración y comunicación de equipos de trabajo que tienen alta movilidad. Es accesible desde cualquier ubicación y tiene disponibles distintos planes en función del tamaño de la empresa, sería un equivalente en la nube a lo que representa la unión de SharePoint y Yammer.

(Torres N., 2014) ejemplifica la implantación de redes sociales propias:

- El Patio, plataforma colaborativa interna de ING BANK, para sus empleados que funciona como cualquier red social. Permite crear comunidades, unirse a ellas según los intereses particulares de cada uno, seguir a otros profesionales del banco, y compartir documentos, vídeos, imágenes o enlaces a webs externas. De esta forma, El Patio se convierte en un canal de comunicación más con los empleados que incluye el componente social, un elemento que lo diferencia del resto. Cualquiera puede generar o compartir información de interés. Esta característica introduce también un cambio cultural en la manera de acceder a la información de los profesionales, ya que

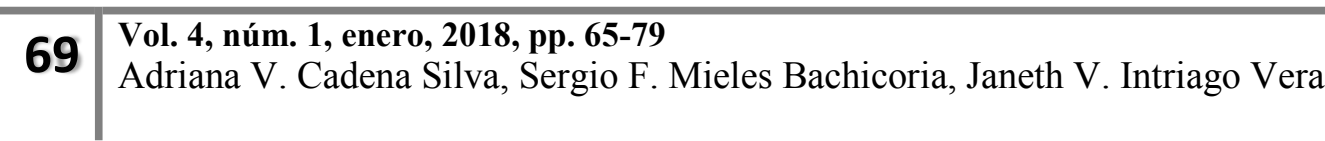


Aproximación al uso de las redes sociales en internet desde una revisión teórica

tienen un rol completamente activo: todos pueden compartir información, intereses y opiniones de manera abierta e inmediata.

- ONE, Grupo AXA, permite a los empleados comunicarse, innovar, interactuar y compartir conocimiento. Esta herramienta 2.0 permite crear redes de contactos, comunidades, wikis y otras funcionalidades de las redes sociales, e identificar perfiles con potencial digital, ya que los empleados más activos son más visibles y surgen de manera espontánea. De este modo, ayuda a trabajar en equipo, incluso entre empleados de diferentes países, promueve una cultura colaborativa en la que el aprendizaje continuo es parte del día a día, y fomenta un estilo bidireccional de comunicación colaborativa. En AXA, muchas ideas relacionadas con el negocio han surgido de un blog de ideación (sistema de votos), y los foros son cada vez más la manera con la que se comparten las experiencias o se toman decisiones en equipo.

Lacayo (2015) expone lo que ganan las organizaciones con el empleo de las redes sociales en la comunicación interna:

- Mayor compromiso por parte de los empleados. Las redes sociales permiten mantener una comunicación frecuente con los empleados, incluso si se encuentran en diferentes ciudades o países; compartiendo noticias e información de la organización, lo que genera un sentimiento de pertenencia y como consecuencia un mayor compromiso con la compañía, al sentirse parte de la misma.

- Promueve el conocimiento compartido y el trabajo en equipo. El proceso de comunicación bidireccional que nos brindan las redes sociales, permite compartir información entre los empleados, promoviendo así el trabajo en equipo. El intercambio de conocimiento genera confianza y mejora la efectividad en los grupos de trabajo.

- Mejora la imagen corporativa: El hecho de estar a la vanguardia de la tecnología constituye una ventaja competitiva para la organización, para lograr que los empleados se sientan más motivados y comprometidos con la cultura organizacional. 
Aproximación al uso de las redes sociales en internet desde una revisión teórica

- Mejora la cultura organizacional: Las redes sociales pueden ser usadas como canal para transmitir los valores y cultura de la organización, lo cual tendrá un gran impacto en los empleados, y asimismo estos podrán transmitirlo a los clientes.

- Mayor claridad de la información: Con frecuencia, suele suceder que los empleados no tienen claridad en la información que se les transmite, por lo tanto sus acciones no van direccionadas hacía los objetivos estratégicos de la organización. Las redes sociales permitirán resolver cualquier duda que tengan los empleados y así obtener retroalimentación de su parte que permitirá plantear acciones de mejora.

- Economía: Mantener una comunicación frecuente y oportuna en grandes organizaciones requiere de una alta inversión económica e implica demasiado tiempo. Las redes sociales permiten gestionar las relaciones con los empleados de manera eficiente y económica. (Lacayo A., 2015)

\section{EI uso de las redes sociales en la mercadotecnia, publicidad y comercio}

Las redes sociales no solo han cambiado la forma en la que las personas se relacionan entre ellas, sino también la forma en la que se relacionan con las empresas y las marcas. De ahí que también el marketing haya sufrido una evolución drástica en los últimos años para adaptarse y sacarle el máximo provecho a ese cambio de hábitos sociales. Primero se empezó a hablar del comercio electrónico o e-

commerce. Pero con el aumento del uso de las TIC, el aumento del ancho de banda, y especialmente con la masificación del uso de las redes sociales se ha generalizado el uso del término "marketing 2.0" para referirse a las nuevas técnicas y a los nuevos ámbitos de uso de las técnicas de marketing. Además, Philip Kotler ya ha ido un paso más allá y ha introducido el término "marketing 3.0". (Sanjaime Calvet L., 2012)

"(...) la participación del usuario en las redes sociales, no solo es un nativo digital, sino que 'es un actor de la conversación' que asume un rol proactivo en situaciones negativas o positivas de la marca, debido a que su interacción puede dar lugar a marketing de dos vías. Entonces el rol del usuario es:

- Participar y compartir sus experiencias

\section{1} Vol. 4, núm. 1, enero, 2018, pp. 65-79 
Aproximación al uso de las redes sociales en internet desde una revisión teórica

- Interactuar con las marcas

- Aportar ideas a la marca

- Actuar como expertos de la marca" (Rojas S.I., s/f)

Rojas (s/f) expone que:

- Las redes sociales se convirtieron en un canal de publicidad y una nueva cultura para los microempresarios porque representan un medio interactivo y potencial que contribuye a su crecimiento en cualquier etapa o ciclo de vida. Parte de ello, se debe a que la información y la investigación de mercado que se puede extraer de este medio, permite reducir costos, conocer las necesidades del consumidor, mejorar las estrategias, generar ideas creativas, entre otras.

- Las redes sociales brindan grandes beneficios a las microempresas, ya que según los datos obtenidos, representan una herramienta económica para hacer publicidad, contribuyen al posicionamiento y mejora la participación y rentabilidad de los negocios. Estos indicadores aumentan las posibilidades comerciales para las marcas en crecimiento, ya que es más costoso pautar en los medios tradicionales.

- Una publicidad bien administrada en las redes sociales, le brinda la posibilidad al empresario de construir una ventaja competitiva para su marca, sin embargo, el éxito va relacionado con la administración, control y moderación los diálogos.

- Pautar en las redes sociales es muy económico y va de la mano con la tecnología y la innovación, por lo que cumple con las expectativas del usuario y del microempresario ya que por un bajo costo puede mantener una presencia en las redes sociales y asegurarse de hacer estudios de mercado demográficos, monitorear las entradas, revisar los comentarios y medir el entorno en general.

Las empresas han decidido trasladar sus puntos de venta a Internet y la han convertido en un nuevo canal de distribución. De ahí que esté cada vez más extendido el uso del comercio electrónico, es decir, la compra-venta de productos y servicios a través de la red. Todos estos factores han propiciado que el marketing haya sufrido una importante evolución durante los últimos años y haya surgido el

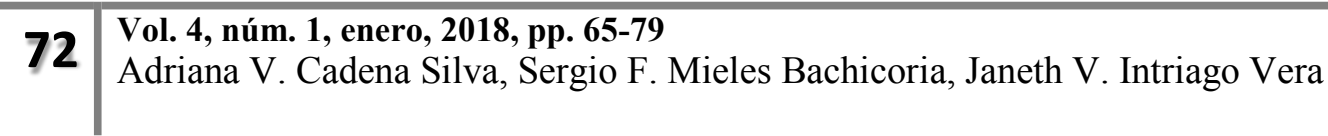


Dom. Cien., ISSN: 2477-8818

Vol. 4, núm. 1, enero, 2018, pp. 65-79

Aproximación al uso de las redes sociales en internet desde una revisión teórica

"marketing online". (Sanjaime Calvet L., 2012) Aquí se incluye el 'marketing 1.0' para el centrado en el producto, el 'marketing 2.0' para el centrado en el cliente y marketing 3.0 para ir más allá, implica conocer sus preocupaciones y sus valores.

Lo que comenzó principalmente como una herramienta dedicada a mejorar la imagen de marca y captar nuevos clientes ha terminado convirtiéndose en un instrumento de venta directa gracias a su correcto desarrollo. Algunas empresas han encontrado en las redes sociales el marco perfecto para desarrollar sus campañas promocionales. Descuentos exclusivos para seguidores, promociones con regalos, sorteos y concursos... Son muchas las vías escogidas para fomentar la compra directa por parte de los "followers" (seguidores) de la compañía y su correcta combinación ha terminado por convertir a las redes sociales en productivos instrumentos de venta para muchas empresas. De ahí la importancia que ha ido tomando en los últimos años el puesto del Community Manager, un perfil profesional que al tiempo que ha ido creciendo en importancia, lo ha hecho en complejidad. Ahora los community managers no son sólo gestores de perfiles sociales que contribuyen a la creación de la imagen de la compañía, son además comerciales encargados de la venta final del producto. (ENAE, 2016)

\section{EI uso de las redes sociales en el sector cultural}

Las redes sociales permiten a las instituciones culturales la interactuación con los usuarios, recoger sus comentarios, conocerlos mejor y establecer una relación duradera. Aprender de sus comentarios para corregir deficiencias, obtener estadísticas de un modo sencillo. Por tanto, el objetivo de usar redes sociales en la cultura es el de crear grandes y fuertes comunidades culturales en las que la institución cultural o las iniciativas culturales sean un catalizador que propicie el intercambio de experiencias. Esto produce dos efectos inmediatos: por una parte, compromiso y cercanía y por otra, difusión, más allá de lo que otros medios de comunicación sean capaces de conseguir y a mucho menor precio. (Peña Aznar J., 2014)

Peña Aznar (2014) desarrolla ejemplos del uso de las redes sociales en el sector cultural, dentro de ellas:

73 Vol. 4, núm. 1, enero, 2018, pp. 65-79

Adriana V. Cadena Silva, Sergio F. Mieles Bachicoria, Janeth V. Intriago Vera 
Aproximación al uso de las redes sociales en internet desde una revisión teórica

- Los concursos son un recurso muy habitual en las redes sociales para aumentar y dinamizar esas comunidades creadas y por su extensión de uso y eficacia merecen un apartado propio.

- Las redes basadas en imágenes como YouTube, Instagram, etc. pueden utilizarse como un modo de co-creación colectiva o también usar otras redes como Twitter o Facebook como inesperados "nuevos espacios" de realización de una obra, ya sea literaria, de teatro o de danza.

- En las redes sociales se comparte entre los seguidores el apoyo que uno ha hecho a una iniciativa cultural, estimulando a otros a participar también. Este efecto de contagio está teniendo un gran impacto para el concepto de "cultura compartida", donde los usuarios encuentran el camino para conseguir que "las cosas se hagan", en especial los proyectos que estaban descartados o parecían imposibles.

Los autores revisados coinciden en que las redes sociales son una gran oportunidad para una institución cultural que tiene la necesidad de difundir sus actividades y crear comunidades alrededor de un tema de interés en común.

El Grupo de Marketing y Capacitación de México expone algunos consejos para la utilización correcta de las redes sociales en el sector de la cultura: (Marketing Capacitación, 2012)

- La cultura digital es indispensable en la institución cultural. Si todos los integrantes del museo, centro cultural o institución cultural no creen en las virtudes de las redes sociales, el esfuerzo será en vano. Para eso, es importante que todos sepan que la relación con el público ya no será unidireccional, sino que tendrán que atender al día las dudas de su comunidad y sus deseos en cuanto a la oferta cultural.

- La publicidad en redes sociales es un gran aliado. Si muchos creen que el trabajo de difusión en Internet no tiene costo, es un gran error. En particular Facebook ofrece varios planes de publicidad que pueden ayudar a hacer crecer la comunidad e informar pertinentemente de sus eventos culturales. El presupuesto que se dedica a la publicidad impresa es siempre muy alto pero no se tiene la posibilidad de medir los resultados. La publicidad en Facebook es flexible según el

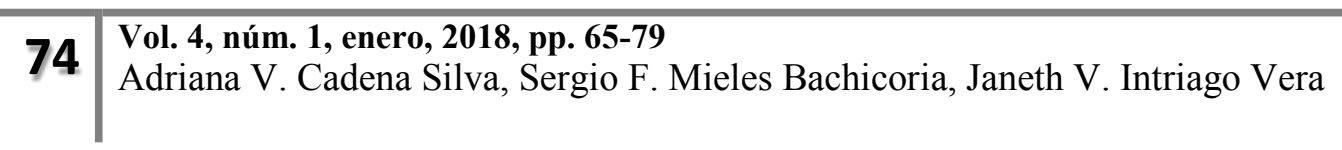


Aproximación al uso de las redes sociales en internet desde una revisión teórica

presupuesto y permite no solo cautivar Fans que recibirán la información de manera regular, se podrá contar con estadísticas precisas para medir el impacto de las campañas.

- Las personas dedicadas a las redes sociales tienen que tener un buen conocimiento de la oferta cultural (...) La persona dedicada a las redes sociales tiene que conocer muy bien la marca, tener excelente ortografía, saber cómo contestar a las preguntas que surgen. El Community manager es la vitrina hacía el mundo exterior, tenemos que elegir con precaución quién será nuestro embajador en las redes sociales.

- Tenemos que contar con un manual de uso para trabajar. ¿Cuál será el estilo de nuestras publicaciones? ¿Hablamos de tú o de Usted? Son preguntas esenciales antes de empezar a incorporarnos a las redes sociales. Tenemos que entender nuestro público, conocer su estilo para acercarnos a nuestra comunidad.

- Las redes sociales son una parte de nuestras campañas de difusión. El trabajo de difusión en redes sociales no está separado de las otras acciones que realizamos en Internet, impresos e inserciones de prensa.

- Tenemos que aprender de los casos de éxito de otras instituciones culturales.

- Desarrollar aplicaciones que permiten llevar la cultura a los usuarios móviles.

\section{El uso de las redes sociales como estrategia de aprendizaje}

La influencia de las redes sociales en educación es importante, teniendo en cuenta que en la actualidad todas las nuevas tendencias e informaciones relevantes en cualquier ámbito sociopolítico, económico y también educativo, se mueve en las redes sociales, siendo básico integrarse en ellas. No solo el profesorado y el alumnado, sino todos los miembros de la Comunidad Educativa deberían estar inmersos en la red. (Pérez García L., 2013)

Las herramientas de la Web 2.0 pueden ser aprovechadas con una ventaja competitiva para trabajar en forma colaborativa, ya que son plataformas gratuitas y accesibles, que favorecen la motivación y

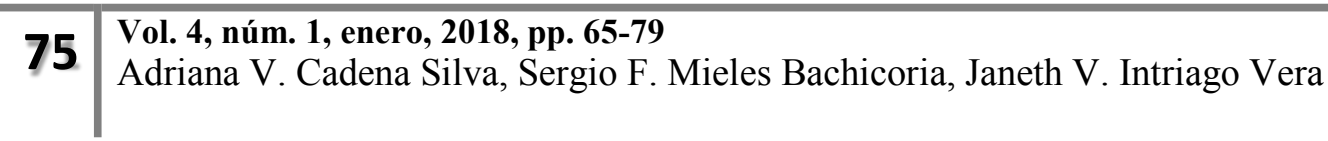


Aproximación al uso de las redes sociales en internet desde una revisión teórica

el interés de los estudiantes al momento de buscar estrategias que beneficien su aprendizaje (...) El uso de las redes sociales en las universidades del mundo ha facilitado la comunicación y el aprendizaje colaborativo, y ha introducido nuevas formas de trabajo entre los actores de los procesos de formación. (Islas Torres C., 2011)

La posibilidad que nos ofrece la red de mantener contacto con personas a las que admiras, o con personas que te pueden aportar documentación en cualquier ámbito de la vida, ha supuesto que las fronteras desaparezcan, haciendo posibles muchas cosas que antes eran impensables. (Pérez García L., 2013)

Meso (2010) demostró, tras trabajar con 130 alumnos, que $87 \%$ de ellos son usuarios activos de los sitios web de relaciones sociales on line, los cuales forman parte de sus prácticas comunicativas usuales, y 66 por ciento se conectan a diario, hábito que se convierte en una cultura de "conexión constante". Las redes sociales más extendidas entre los alumnos de las universidades del País Vasco son: 60 por ciento utilizan Twitter; 14 por ciento, Facebook; 14 por ciento, Fotolog; 8 por ciento, MySpace; 2 por ciento, Messenger; 1 por ciento, Blogger; y 1 por ciento, Hi5. (Meso, 2010)

Islas Torres (2011) hizo un estudio donde el 71 por ciento de los estudiantes señalaron que usan las redes sociales para informar a sus compañeros sobre actividades escolares; 42 por ciento, para jugar; y 90 por ciento, para comunicarse con los demás, lo cual muestra la importancia y fuerza que las redes sociales están tomando en el proceso educativo. Algunos estudiantes eligieron una o más opciones en el mismo cuestionamiento, por lo que pueden utilizar las redes en más de una actividad.

De Haro (2010), señala los beneficios que nos puede aportar una red social en el trabajo con los alumnos:

Permite centralizar en un único sitio todas las actividades docentes, profesores y alumnos de un centro educativo.

Aumento del sentimiento de comunidad educativa para alumnos y profesores debido al efecto de cercanía que producen las redes sociales.

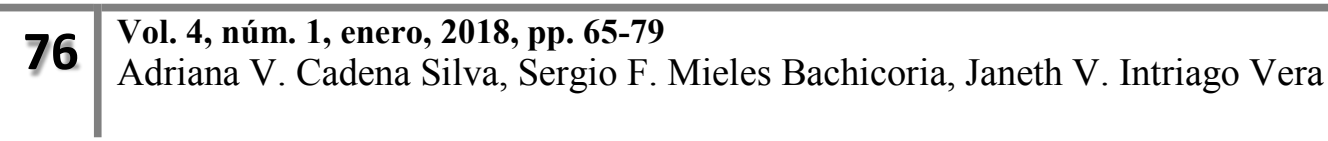


Dom. Cien., ISSN: 2477-8818

Vol. 4, núm. 1, enero, 2018, pp. 65-79

Aproximación al uso de las redes sociales en internet desde una revisión teórica

Mejora del ambiente de trabajo al permitir al alumno crear sus propios objetos de interés, así como los propios del trabajo que requiere la educación.

Aumento en la fluidez y sencillez de la comunicación entre profesores y alumnos.

Incremento de la eficacia del uso práctico de las TIC, al actuar la red como un medio de aglutinación de personas, recursos y actividades. Sobre todo cuando se utilizan las TIC de forma generalizada y masiva en el centro educativo.

Facilita la coordinación y trabajo de diversos grupos de aprendizaje (clase, asignatura, grupo de alumnos de una asignatura, etc.) mediante la creación de los grupos apropiados.

Aprendizaje del comportamiento social básico por parte de los alumnos: qué puedo decir, qué puedo hacer, hasta dónde puedo llegar, etc. (De Haro J.J., 2010)

Las redes sociales, y en general las herramientas de la Web 2.0, pueden considerarse una estrategia de aprendizaje, porque tienen el papel de facilitadoras de información y medios para la integración y comunicación; de forma voluntaria, los estudiantes accederían a ellas y darían un valor agregado a sus procesos de aprendizaje. (Islas Torres C., 2011)

\section{Conclusiones}

Cada vez se expande con mayor intensidad una vida digital que impulsa a las organizaciones a sumergirse en las nuevas formas de comunicación por medio de Internet. Las organizaciones deben adaptar su cultura y metodología para sacar partido al gran potencial que ofrecen las redes sociales. Este trabajo muestra cómo existen diferentes iniciativas en distintos ámbitos en cuanto al uso de las redes sociales.

No obstante a pesar del uso indiscutible ventajoso de las redes sociales, también en la literatura se abordan sus desventajas entre ellas: la distracción que puede generar, la variedad de información irrelevante, la pérdida del contacto personal, el uso abusivo, compulsivo o adictivo. Esto es importante tenerlo en cuenta.

77 Vol. 4, núm. 1, enero, 2018, pp. 65-79
Adriana V. Cadena Silva, Sergio F. Mieles Bachicoria, Janeth V. Intriago Vera 
Aproximación al uso de las redes sociales en internet desde una revisión teórica

\section{Referencias bibliográficas}

Área Moreira M. (2008). La innovación pedagógica con TIC y el desarrollo de las competencias informacionales y digitales. Investigación en la Escuela, 64, págs. 5-17.

Cristófol F.J. (2016). Las redes sociales en la empresa: cuatro beneficios para la comunicación interna. Obtenido de http://www.eleconomista.es

De Haro J.J. (2010). Las redes sociales en educación. Obtenido de http://jjdeharo.blogspot.com.es/2008/11/la-redes-sociales-en-educacin.html.

ECURED. (2017). Internet. Obtenido de www.ecured.cu

ENAE. (2016). La importancia de las redes sociales en el Marketing Digital. Obtenido de http://www.enae.es/users/enae

Islas Torres C., C. A. (2011). Uso de las redes sociales como estrategias de aprendizaje. ¿Transformación educativa? Apertura vol. 3, núm. 2.

Lacayo A. (2015). Las redes sociales como herramienta para gestionar la comunicación interna en las organizaciones. Obtenido de http://reportedigital.com/transformacion-digital/redes-socialesherramienta-para-gestionar-comunicacion-interna-organizaciones/

Marketing Capacitación. (2012). Redes sociales y difusión de la cultura. Obtenido de https://www.marketingcapacitacion.com

Peña Aznar J. (2014). ¿Sirven para algo las redes sociales en el sector cultural? Anuario.

Pérez García L. (2013). Redes Sociales y Educación. Creatividad y Sociedad.

PYMES. (2012). Las redes sociales como herramienta de mejora en la comunicación interna de las empresas. Obtenido de http://www.bbvacontuempresa.es/pymes

Rojas S.I. (s/f). La publicidad en las redes sociales: impulso a las microempresas en crecimiento. 
Dom. Cien., ISSN: $2477-8818$

Vol. 4, núm. 1, enero, 2018, pp. 65-79

Aproximación al uso de las redes sociales en internet desde una revisión teórica

Sanjaime Calvet L. (2012). Redes sociales y marketing. Escola Tècnica Superior d'Enginyeria Informàtica Universitat Politècnica de València.

Torres N. (2014). Se consolida el uso de las Redes Sociales como canal de comunicación interna en la empresa. Obtenido de http://www.observatoriorh.com

79 Vol. 4, núm. 1, enero, 2018, pp. 65-79

Adriana V. Cadena Silva, Sergio F. Mieles Bachicoria, Janeth V. Intriago Vera 